\title{
Norois
}

Environnement, aménagement, société

$202 \mid 2007 / 1$

Recomposition des espaces ruraux

\section{La ruralité entre les appréciations statistiques et les représentations sociales : comprendre la reconfiguration socio-spatiale des territoires ruraux québécois}

Rurality between statistical appraisal and social representations: understand the socio-spatial restructuration of Quebec rural territories

\section{Bruno Jean et Stève Dionne}

\section{OpenEdition} Journals

\section{Édition électronique}

URL : http://journals.openedition.org/norois/1599

DOI : 10.4000/norois. 1599

ISBN : 978-2-7535-1550-5

ISSN : 1760-8546

Éditeur

Presses universitaires de Rennes

\section{Édition imprimée}

Date de publication : 1 mars 2007

Pagination : 9-19

ISBN : 978-2-7535-0457-8

ISSN : 0029-182X

Référence électronique

Bruno Jean et Stève Dionne, «La ruralité entre les appréciations statistiques et les représentations sociales : comprendre la reconfiguration socio-spatiale des territoires ruraux québécois », Norois [En ligne], 202 | 2007/1, mis en ligne le 01 mars 2009, consulté le 19 avril 2019. URL : http:// journals.openedition.org/norois/1599; DOI : 10.4000/norois.1599 


\title{
LA RURALITÉ ENTRE LES APPRÉCIATIONS STATISTIQUES
}

ET LES REPRÉSENTATIONS SOCIALES : COMPRENDRE

\section{LA RECONFIGURATION SOCIO-SPATIALE DES TERRITOIRES RURAUX QUÉBÉCOIS}

\author{
Bruno Jean, Stève Dionne \\ Centre de Recherche sur le Développement Territorial - Université du Québec (Rimouski), \\ 300, allée des Ursulines - CP 3300 Rimouski (Québec) Canada G5L 3Al \\ bruno_jean@uqar.qc.ca,steve_dionne@uqar.qc.ca
}

\begin{abstract}
RÉSUMÉ
La classique distinction rural/urbain est moins éclairante pour comprendre la ruralité moderne au Québec que l'accentuation de ses différenciations internes. Cette variance pose la question de la meilleure approche typologique pour comprendre des nouvelles reconfigurations socio-spatiales de la ruralité et rendre intelligibles les facteurs responsables d'une telle diversification. Cet article propose une réflexion sur les transformations des différentes ruralités au Québec et sur l'évolution des représentations qui leur sont associées dans l'espace public.
\end{abstract}

MotS CLÉ : Recomposition - Représentations - Ruralité - Territoire - Typologie.

\begin{abstract}
Rurality between statistical appraisal and social representations: understand the socio-spatial restructuration of Quebec rural territories

To understand modern rural spaces in Quebec the traditional distinction between rural and urban is less useful than the increasing internal differentiations inside of these rural areas. These variations raise the question of the best typological approach to understand new socio-spatial reconfigurations of the rural spaces and to make the factors responsible for such a diversification understandable. A study of the transformations of various rural spaces in Quebec and on the evolution of the representations which are associated with them in public space is discussed in this paper.
\end{abstract}

KEY WORDS : Representation - Restructuration - Rural Spaces - Territory - Typology.

La ruralité caractérise le quart de la population du Québec et plus de 80 \% du territoire habité, sans parler de la contribution des ressources à la prospérité économique d'ensemble. L'histoire et la géographie se sont conjuguées avec les transformations de l'économie pour façonner une variété de régions rurales relativement différenciées. Cette diversité est devenue telle que l'on peut soutenir l'hypothèse que la classique distinction rural/urbain est dorénavant moins fondamentale et moins éclairante pour comprendre la ruralité moderne au Québec que l'accentuation des différenciations 
internes de cette même ruralité. Cela pose donc la question de la meilleure approche typologique pour rendre compte de cette évolution et rendre intelligibles les facteurs responsables d'une telle diversification des espaces ruraux.

Parallèlement, l'approche constructiviste nous invite à appréhender les réalités sociales comme la ruralité, d'abord en tant que constructions sociales, c'est-à-dire de représentations qu'il faut étudier comme telles. Car cette ruralité plurielle, repérable dans l'espace, est également une réalité idéelle qui s'exprime par une symbolique essentiellement représentative et discursive. Et si la construction d'une typologie actualisée des ruralités doit répondre à un besoin de connaissance de la diversité de la réalité rurale, cet exercice doit aussi intégrer la caractérisation des grands types de représentations qui la définissent, sur la base des discours qui structurent le champ de ces représentations, à savoir le discours social, le discours politique et le discours savant. Un certain discours social sur la ruralité, par exemple, fait passer celle-ci de quelque chose qui est traditionnel et appelé à disparaître ou à changer avec le déploiement de la modernité, à une représentation de cet objet comme un environnement, ou des paysages qui sont des «biens publics » ou collectifs qui n'appartiennent pas seulement aux ruraux mais à toute la société. Cette nouvelle représentation d'un type de ruralité devient potentiellement porteuse d'incompréhension et de conflits de cohabitation tout autant qu'elle peut permettre de créer de nouvelles solidarités rurales-urbaines basées sur une meilleure compréhension des liens d'interdépendance entre ces deux mondes.

En prenant en partie appui sur une recherche récente des auteurs, interpellés pour mesurer les effets de la récente politique rurale québécoise, l'objectif de cet article est de présenter l'état d'une réflexion en cours sur les transformations des différentes ruralités au Québec et l'évolution des représentations qui leur sont associées dans l'espace public. À la base de cet exercice, nous souhaitons savoir comment territoires et représentations permettent-ils de légitimer autant de types de ruralités?

\section{Les visages nombreux et singuliers de la ruralité}

Dans notre compréhension, l'accroissement des différenciations internes au monde rural représente un trait décisif de l'évolution de la ruralité québécoise depuis un demi siècle.

\section{UNE RURALITÉ QUI A BEAUCOUP CHANGÉ}

Rappelons qu'au Québec, la ruralité d'autrefois était fortement homogène sur le plan culturel et économique, du moins était-elle essentiellement différenciée en quelques types facilement repérables. Jusque dans la première moitié du $\mathrm{XX}^{\mathrm{e}}$ siècle, on pouvait dire que le monde rural correspondait à un semis de paroisses plus ou moins semblables les unes aux autres. D'autres observateurs mettaient l'accent sur la différenciation entre les vieilles paroisses riveraines du Saint-Laurent avec une culture agraire profonde, et les nouvelles paroisses de colonisation agro-forestières où se formait un nouveau type social : le colon qu'on opposait à l'habitant des basses terres.

Or, ces espaces ruraux ont été le théâtre de profondes transformations, spécialement au cours des six dernières décennies. Dans l'immédiat après-guerre, des bouleversements considérables ont affecté une agriculture et une foresterie qui avaient traditionnellement caractérisé le travail en milieu rural et historiquement fondé la rationalité de l'occupation du territoire. Rapidement, le nombre de fermes et la population agricole ont fondu sous les effets conjugués d'une concentration des exploitations (les fermes commerciales devenant de plus en plus grandes et capitalisées) et d'une concentration spatiale de l'agriculture (déprise agricole, recul des superficies cultivées, voire disparition de l'agriculture dans maintes localités). De même, la professionnalisation et la mécanisation du travail et des opérations en forêt, la surexploitation ou le déplacement des sites d'exploitation toujours plus loin, ont contribué sinon à faire disparaître l'orientation forestière de nombreuses communautés, du moins à réduire considérablement le nombre d'emplois associés à cette filière économique fondatrice. 
Toutes ces transformations en milieu rural se sont déroulées pendant une période d'explosion démographique et de croissance générale de l'économie industrielle et urbaine (les Trente glorieuses). La population rurale, de plus en plus mobile avec la généralisation de l'automobile et pénétrée des valeurs urbaines avec le développement de la consommation et des médias de masse, a connu un élargissement de l'éventail des possibilités individuelles. Pour les jeunes ruraux de cette époque, la décision d'émigrer devenait alors irrésistible dans ce contexte économique partout ailleurs favorable.

Quant aux dernières décennies, la tertiarisation de l'économie a modifié la structure spatiale des économies rurales, c'est-à-dire la composition et la répartition des équipements de l'offre commerciale et des services publics. L'accroissement toujours plus important de la mobilité individuelle, avec pour conséquence un élargissement net de la fonction résidentielle des territoires ruraux, de même que les implications du passage d'une économie des ressources à une économie du savoir entraînant reconversion industrielle et nouvelle spécialisation des économies régionales, ont convergé et contribué à pousser plus avant un processus de concentration des fonctions et de différenciation des espaces ruraux entre petites villes et gros villages en croissance d'un côté, et petites localités périphériques en déclin sévère de l'autre. Ils ont favorisé en quelque sorte une érosion différentielle de la population rurale.

\section{LA RECONFIGURATION SOCIO-SPATIALE DE LA RURALITÉ}

En étudiant les processus à l'œuvre dans la reconfiguration socio-spatiale de la ruralité québécoise (mais également canadienne), force est de constater deux choses. D'abord, que ce rural perdure et qu'environ le quart de la population continue de faire l'expérience quotidienne des deux plus robustes critères définissant la ruralité : un type particulier de rapport à l'espace, redevable à la faible densité de population (la mobilité, critère géographique), et un type particulier de sociabilité, redevable à la faiblesse des effectifs démographiques (l'interconnaissance, critère sociologique).

De même, toutes les appréciations statistiques actuelles de la réalité conduisent à des constats généraux similaires : des milieux ruraux sont encore en déstructuration ici, mais d'autres sont en pleine croissance ailleurs. Ils peuvent être toujours basés sur les ressources primaires ici, ou exportateurs de haute technologie ailleurs. Des territoires ruraux agricoles sont retournés en quelques décennies à une économie essentiellement forestière ici alors que d'autres, ailleurs, ont vu l'intensité de leur activité agricole multipliée. En observant l'évolution des structures par âge et de peuplement, on s'aperçoit que des territoires ruraux entiers, à bonne distance des grandes villes mais sans en être trop déconnectés, jadis forestiers puis devenus hauts lieux de villégiature à partir des années 1960-1970, sont maintenant en train de devenir des milieux ruraux principalement résidentiels, fondés pour beaucoup sur des aménités de paysage et contribuant à définir un nouveau type de ruralité (nous pourrions dire : une nouvelle expérience de ruralité) encore totalement inexistant il y a cinquante ans. Et nous pourrions ainsi continuer d'aligner des exemples.

En fait, ce qu'il importe de retenir de ces transformations dans le contexte québécois, c’est que la ruralité offre aujourd'hui des visages nombreux et singuliers, et que les statistiques montrent maintenant depuis une quinzaine d'années, qu’à côté des cas de localités en décroissance continue de population, on repère également de nombreuses situations de stabilisation démographique, voire de reprise de la croissance et de fort développement. Les travaux d'Augustin Épenda (2003) qui s'est intéressé spécifiquement à la mesure du niveau de développement des municipalités rurales québécoises ont permis de dégager une conclusion fort intéressante en appui à cette idée. Il s'est appuyé sur trois indices différents bien usités : indice global de développement du ministère des régions, indice de développement humain modifié de l'ONU, indice de défavorisation de Pampalon.

C'est ainsi que son identification de seuils sur la distribution de fréquence des municipalités rurales classées par indice selon leur niveau de développement a fait continuellement ressortir, quelque soit l'indice choisi, que les localités défavorisées ou en dévitalisation comptaient toujours, 
de façon stable, pour environ $20 \%$ des effectifs. Quelle que soit la mesure utilisée, il observe peu de variation des effectifs de cette « dévitalisation rurale » (une localité sur cinq, ce qui est beaucoup mais tout de même loin de ce que l'on peut lire de certains discours inquiétants sur la dévitalisation du «monde rural » et la « désintégration des régions »). La composition du groupe des municipalités rurales les plus fragiles est constante et, que l'on s'y prenne d'une manière ou d'une autre en interchangeant des variables, les mesures de la dévitalisation rurale obtenues sont équivalentes, elles donnent toujours à peu près les mêmes résultats.

\section{UNE RÉALITÉ NÉANMOINS MAL COMPRISE}

Pendant longtemps, les études rurales se sont attachées à comprendre les écarts de développement entre les espaces ruraux et urbains. Plusieurs travaux ont montré une réduction des disparités entre ces milieux, tant au niveau des modes de vie que des conditions de vie (revenus, emplois, etc.), alors que d'autres recherches ont illustré des différences persistantes. Pour nous, sans juger la pertinence de ce genre de travaux, de toute manière rendus épineux par la difficulté d'opposer en bloc le rural à l'urbain, ce qui représente un trait décisif de l'évolution de la ruralité des trente (voir des soixante) dernières années, c'est un accroissement des différenciations internes de la ruralité elle-même.

Nous acceptons l'idée selon laquelle, à maints égards, le rural et l'urbain présentent dans l'ensemble de moins en moins de différences notables, et que la traditionnelle et commode opposition ville/campagne est de moins en moins significative et éclairante pour comprendre la ruralité dans la modernité avancée. Non seulement la ruralité québécoise n’est pas unidimensionnelle et homogène sur le vaste espace habité, mais nous croyons même que sa diversité est l'un des aspects les plus mal maîtrisés et qui souffre le plus d'une actualisation des connaissances. C'est pour cela qu'il nous apparaît désormais comme un défi scientifique des études rurales au Québec, de proposer une typologie des territoires capable de rendre compte de ce processus historique de reconfiguration socio-spatiale de la ruralité.

À ce défi correspondent néanmoins quelques précédents. Les auteurs ont ainsi été amenés à recenser une dizaine de typologies rurales (Jean et al., 2004) depuis celle de Gérald Fortin qui, en 1960, faisait le portrait socioéconomique des municipalités du Québec en cartographiant les milieux agricoles prospères, les milieux agricoles moyens ou marginaux et les localités pauvres. Fortin esquisse également dans son exercice typologique les territoires de la ruralité forestière distinguant celle des «forêts concédées » de celle des forêts «non concédées » (par xemple les localités dont l'économie reposait principalement sur l'exploitation de la forêt privée de celles localisées sur les pourtours de l'écoumène, royaume des grandes compagnies forestières qui ont fait un temps la prospérité du Canada).

Dans plusieurs autres cas, quelles que soient les variables utilisées, les exercices typologiques reposent sur une caractérisation du degré de proximité ou d'éloignement des agglomérations métropolitaines. De l'examen de ces diverses esquisses typologiques, il ressort que les grandes tendances lourdes qui concernent la structuration et le développement territorial au Québec sont relativement stables.

Cependant, le problème général demeure le suivant : il existe peu de synthèses d'envergure sur ce phénomène de reconfiguration spatiale de la ruralité québécoise, de recomposition des systèmes ruraux pouvant rendre intelligibles les nouvelles réalités rurales (par exemple : milieux en véritable déstructuration, milieux essentiellement agricoles prospères mais sous-peuplés, milieux forestiers stagnants, milieux agro-forestiers de plus en plus touristiques, localités rurales en croissance du fait de leur polarisation des activités commerciales et de services, etc.). Nous croyons que la préoccupation à l'égard de la persistance et des transformations de la ruralité, la demande d'une telle vision renouvelée des réalités rurales à travers une typologie adéquate, répondrait autant à une exigence théorique qu'à une demande sociale, celle exprimée par les ruraux eux-mêmes. Il reste d'ici là à déterminer la meilleure approche typologique qui permettrait de rendre compte de 
cette évolution et rendre intelligibles les facteurs à l'origine d'une telle diversification des milieux ruraux contemporains au Québec.

Cet objectif n'est pas une fin en soi, il permet selon nous de mieux saisir le rôle et l'importance des caractéristiques empiriques les plus déterminantes de divers types de territoires ruraux qui influencent, donc rétrécissent, l'éventail des représentations possibles. Car les faits de géographie (économique, historique, culturelle, etc.) ne déterminent pas, indépendamment de tout, ce que sont les diverses ruralités québécoises contemporaines. Ils ne font que participer en substrat à la « construction sociale » de ces réalités car les individus et les groupes ne peuvent pas construire de telles catégories sans ancrage empirique, sans correspondance avec les contingences des réalités matérielles sur lesquelles s'édifient les identités.

\section{La ruralité : une construction sociale en perpétuelle évolution}

Devant l'énorme difficulté de définir ce qu'est le rural pour des fins de mesure, les statisticiens ont convenu d'identifier la ruralité par la négative en convenant qu'elle est ce qui n'est pas urbain, une réalité en apparence plus facile à circonscrire. En faisant une telle opération, la statistique illustre aussi le fait qu'elle élabore des appréciations prétendument objectives mais qui ne sont pas sans liens avec les représentations sociales de la réalité rurale, lesquelles peuvent évoluer dans le temps. Une réalité sociale comme la ruralité n'existe pas en dehors des processus discursifs de construction sociale de cet objet en réalité signifiante. La ruralité est donc une construction sociale et il s'agit alors de comprendre comment cette réalité est produite socialement.

L'étude des représentations de la ruralité se présente comme un vaste chantier de recherche dont on connaît encore mal l'ampleur. Ces représentations sont aujourd'hui multiples et on peut raisonnablement penser qu'une représentation peut dominer le champ sociopolitique et devenir ainsi une référence incontournable. Un premier constat est plutôt gênant : les représentations se cristallisent dans des discours, or, les ruraux, pendant longtemps, n'ont pas été des gens de parole... mais plutôt des sans voix. Ce sont d'autres, comme parfois une certaine petite bourgeoisie libérale souvent issue de son sein, qui ont pris la parole, qui ont écrit et alors façonné des images de cette ruralité dont ils prétendaient connaître ou défendre les intérêts.

S’il faut distinguer le discours urbain contemporain sur la ruralité du discours des ruraux euxmêmes, il faut aussi voir les rapports entre trois grands types de discours (tableau 1) qui participent de la construction des représentations contemporaines de la ruralité, à savoir le discours social (qui se structure dans les médias et qui alimente une opinion publique), le discours scientifique (qui essaie de se donner des critères objectifs mais qui reste passablement vulnérable aux diktats des concepts à la mode et aux idéologies du moment) et le discours politique (qui tente de prendre appui à la fois sur le discours social et sur le discours scientifique).

Avec le passage non pas à une prétendue post-modernité mais à ce que Antony Giddens appelle la modernité avancée, les représentations antérieures de la ruralité deviennent plus claires, plus saisissables. Au risque de pécher par simplification abusive, nous avons tenté de faire une cartographie conceptuelle des grandes représentations dans ces trois types de discours : le discours sociologique, le discours social et finalement le discours politique ${ }^{1}$.

\section{LE DISCOURS SOCIOLOGIQUE}

Dans le cas du discours sociologique, il est apparu nécessaire de distinguer la tradition intellectuelle européenne et la tradition nord-américaine. Dans cette dernière, c'est sans soute le concept de folk society qui caractérise le plus la pensée sociologique sur la ruralité à l'ère de la modernité. Le monde rural est pour Robert Redfield une folk society, une société traditionnelle.

1. Une première version de cette réflexion sur les discours ruraux a été publiée dans un chapitre d'un ouvrage sur les paysages ruraux. Voir : (Jean, 2003). Voir aussi : (Jean, 2004). 
Avec la généralisation de la modernité, ces folk societies sont appelées à devenir autre chose : soit disparaître, soit devenir des sociétés urbaines. On retrouve ici le grand paradigme sociologique de l'évolutionnisme où la ruralité, associée au passé, à la tradition, est destinée à disparaître avec la modernité. Il faut ajouter ici que ce concept tire en partie son origine de la distinction entre la Gemenschaft (communauté) et la Gessellschaft (société) de l'allemand Ferdinand Tonniës.

Dans la variante européenne du discours sociologique, on ne retrouve pas cette idée de ruralité comme une société traditionnelle, voir pratiquement primitive, destinée à disparaître. Ces traits sont bien sûr régulièrement évoqués mais ce qui semble dominer l'analyse sociologique, c'est l'association entre le rural et la paysannerie, avec les notions de sociétés paysannes, de communautés paysannes. Une société paysanne est une société complexe, organisée, mais selon des modes de régulation qui sont mis à mal avec la modernité et qui disparaissent complètement avec la modernité avancée. C'est cette variante européenne qui va donner une couleur décisive à la sociologie rurale comme une sociologie d'un groupe particulier, les paysans, qui dominent effectivement les sociétés agraires d'autrefois, devenus des agriculteurs inscrits dans des rapports marchands dans les sociétés modernes. Nous hériterons de cette histoire intellectuelle d'une sociologie rurale qui est pratiquement dédiée à l'étude des transformations d'un seul secteur socio-économique de la ruralité, l'agriculture. Avec la modernité, cela devient gênant car l'agriculture n'est plus une activité dominante dans son propre espace, son propre environnement.

La place nous manque ici pour expliquer les fondements de cette conception relativement différenciée mais dont la construction renvoie très certainement à des histoires et des identités assez différentes. Il n'est donc pas étonnant que le concept de société paysanne soit européen, l'Amérique du Nord comme du Sud n'ayant probablement pas fait l'expérience de telles organisations sociales. Par ailleurs, la notion de folk society ne pouvait apparaître que dans une société tout entière vouée à une entreprise de modernisation urbanisante et industrialisante, se devant alors de faire apparaître la ruralité comme un anachronisme. Si cette différenciation entre une sociologie rurale américaine et européenne, pour ne pas dire étatsunienne et française, est intéressante, l'évolution du discours sociologique avec l'entrée des sociétés dans la modernité avancée l'est encore plus.

Du coté américain, la représentation dominante du rural dans la littérature en sciences sociales est très nettement celle du «non metropolitan area ». Un rapide coup d'œil dans les articles d'une revue comme Rural Sociology ou dans les titres des communications de la Rural Sociology Society, depuis plus de vingt ans, en témoigne avec éloquence. La ruralité qui intéresse les chercheurs « ruralistes $^{2}$ » américains est celle qui concerne les populations vivant dans ces espaces, ces territoires en dehors des métropoles, des grandes villes et de leurs aires d'influence. La représentation du rural, plutôt sociale avec la folk society devient une représentation fortement spatiale avec le «non metro adjency».

Dans cette représentation, les maillons inférieurs de la hiérarchie urbaine sont aussi associés au rural. Dans plusieurs universités, on voit des centres de recherche ruraux où l'objet est défini comme le "Rural and Small Towns». Une telle conception a l'avantage de nous rappeler l'importance de la dimension écologique ou environnementale des milieux de vie; vivre dans un milieu fortement urbanisé ne signifie pas la même chose que vivre en campagne ou dans une petite ville qui en est son émanation directe. Par contre, elle élude plusieurs problèmes théoriques sur le statut de la ruralité dans la modernité avancée. Généralement, cette sociologie rurale américaine est plutôt une sociologie descriptive des problèmes sociaux des populations vivant dans les milieux non métropolitains.

2. Notons ici que la notion française de «ruraliste» ne fait pas sens dans la langue anglaise étatsunienne comme dans la langue française québécoise. Comme le ruralisme y désigne un idéologie rétrograde (et des idées politiques plutôt très à droite), les intellectuels n'y peuvent s'autoproclamer ruralistes comme on peut le voir avec l'ARF en France (Association des ruralistes français). 
Pendant ce temps, on a vu le discours sociologique européen glisser progressivement vers le rural comme environnement (Mathieu, Jollivet, 1989). Le nouveau champ des études environnementales a été passablement investi par les ruralistes. Et la dimension environnementale prend une importance particulière (et en ce sens la différence avec l'évolution de la pensée sociologique ruraliste américaine n'est pas si diamétralement opposée). Cette sociologie rurale européenne, après s'être longuement intéressée à l'urbanisation des campagnes (un thème de recherche majeur qui va d'ailleurs provoquer une crise au sein même des études rurales), rappelle maintenant que la seule dimension écologique pèse de tout son poids. Le cadre de vie devient une sorte de variable décisive, discriminante. Et comme le dit si bien Marcel Jollivet, les ruraux redeviennent dans ce contexte des acteurs sociaux importants car la tâche de la protection de la nature se joue principalement en campagne. Qui plus est, la protection de l'environnement est devenue un enjeu central qui intéresse toute la société. Une chose si importante ne peut plus «être abandonnée » au bon vouloir des seuls ruraux, pas toujours capables de prendre les bonnes décisions en cette matière devenue d'intérêt pour l'ensemble de la société.

Ce qui est commun à cette évolution du discours sociologique sur la ruralité avec l'entrée de nos sociétés dans la modernité avancée, c’est qu'il semble y avoir un consensus sur la disparition des sociétés rurales ou paysannes comme société globale. La ruralité subsiste mais on est devant un vide ou une hésitation pour la caractériser. On s'est rabattu sur la dimension spatiale ou environnementale. Cela nous semble une position à la fois intéressante en ce qu'elle redonne une place à la nature, à l'environnement dans la théorie sociologique, une nécessité comme le souligne si bien les travaux de Bernard Kalaora (1998). Pour plusieurs spécialistes des études rurales contemporaines, voilà une nouvelle chance pour les campagnes. Car au moment où l'on doute que la ruralité ne porte réellement certains caractères socio-économiques distincts, la définition de la campagne comme environnement la promet à une nouvelle sollicitude des pouvoirs publics tout en rappelant l'urgence d'une approche interdisciplinaire élargie conviant les sciences naturelles et les sciences sociales dans l'étude commune des réalités rurales et des questions relatives à son aménagement.

Un nouveau défi pour les sciences sociales se trouve ainsi lancé, celui de replacer la nature dans une théorie de la société. Mais cette position est un peu faible car les dangers d'un repli vers une géographie déterministe, rejetée par la géographie contemporaine elle-même, laissent entière la question d'une solide construction de la ruralité de la modernité avancée en tant qu'objet sociologiquement construit.

\begin{tabular}{|c|c|c|c|c|}
\cline { 2 - 4 } \multicolumn{1}{c|}{} & \multicolumn{4}{c|}{ Discours } \\
\cline { 2 - 3 } \multicolumn{1}{c|}{} & Américain & Souropéen & Social & Politique \\
\cline { 2 - 5 } $\begin{array}{c}\text { Dans la } \\
\text { modernité } \\
\text { naissante }\end{array}$ & $\begin{array}{c}\text { Ruralité }=\text { folk } \\
\text { societies }\end{array}$ & $\begin{array}{c}\text { Ruralité }= \\
\text { communautés } \\
\text { paysannes }\end{array}$ & $\begin{array}{c}\text { La ruralité est } \\
\text { traditionnelle, } \\
\text { dépassée et en } \\
\text { retard }\end{array}$ & $\begin{array}{c}\text { Modernisation } \\
\text { (aménagement rural) }\end{array}$ \\
\hline $\begin{array}{c}\text { Dans la } \\
\text { modernité } \\
\text { avancée }\end{array}$ & $\begin{array}{c}\text { Ruralité = régions } \\
\text { non métropolitaines } \\
\text { (non metro adjency) }\end{array}$ & $\begin{array}{c}\text { Ruralité }= \\
\text { environnement }\end{array}$ & $\begin{array}{c}\text { Le rural comme } \\
\text { paysage valorisé et } \\
\text { environnement }\end{array}$ & $\begin{array}{c}\text { Préservation } \\
\text { (développement } \\
\text { durable) }\end{array}$ \\
\hline
\end{tabular}

Tableau 1 : L'évolution du discours sociologique, social et politique dominant

Evolution of dominant sociological, social and political discourses

\section{LE DISCOURS SOCIAL}

Le discours social dominant se transforme aussi avec le passage de la modernité à la modernité avancée. Bien qu'il soit téméraire de penser qu'il y ait un seul discours social dominant sur le 
monde rural, contrairement au discours sociologique où nous avons distingué deux paradigmes, ici nous n'en présentons qu'un seul sachant qu'une analyse plus approfondie de ses variantes en ferait certainement apparaître d'autres. À l'époque de la modernité naissante, principalement durant la période suivant l'après Seconde Guerre mondiale, la ruralité apparaissait comme un monde qui n'avait pas encore atteint le stade de la modernité caractérisée par la pensée rationnelle, l'urbanisation et l'industrialisation. Le monde rural était donc un monde en retard, traditionnel et dépassé en quelque sorte; un anachronisme qui devra s'ajuster avec le temps par une nécessaire urbanisation des campagnes déjà annoncée par les intellectuels.

Cette dualité rural/urbain, repensée comme la dichotomie traditionnelle/moderne faisant écho au discours sociologique, avait quelque chose de réconfortant. Le moderne urbain l'était d'autant plus qu'une ruralité traditionnelle tardait à disparaître, survivait ça et là au sein de sociétés devenues modernes et urbaines, et confortait donc en cela cette identité nouvelle. La « rencontre de ces deux mondes ${ }^{3}$ » permettait donc à chacun de s'identifier car on sait bien que le processus de construction de l'identité sociale se fait tout autant par la mesure des différences dans l'opposition à l'autre que dans l'affirmation forte d'une appartenance ou d'une référence commune.

Cette posture de la pensée sociale reproduit le même dilemme que celle de la pensée sociologique; si le rural est dépassé, comment peut-on être rural à l'ère de la modernité et plus encore à l'ère de la modernité avancée? Avec la modernité, la ruralité est condamnée! Faisant écho à certains classiques de la littérature sociologique annonçant pourtant la fin d'une certaine ruralité et non de la ruralité comme telle, la pensée sociale dominante est incapable de penser le rural dans la modernité. La ruralité va soit disparaître, soit être intégrée dans le monde moderne en devenant des espaces urbanisés de moindre densité, à un degré plus faible sur le gradient de l'urbanisation modernisante. Pourtant, le monde rural, dans une société urbaine, n'est pas un espace en attente d'urbanisation ou de désertification, mais un territoire avec une vie socioéconomique spécifique et difficilement réductible aux dynamiques urbaines.

Avec l'avènement de la post-modernité, mieux nommée modernité avancée selon les termes de Giddens, il s'est produit un curieux renversement de perspective. Ce monde rural un tantinet traditionnel et passéiste a été assez rapidement l'objet d'une survalorisation par les masses urbaines y découvrant là un monde de l'authenticité. Le rural est toujours le monde du commencement, des origines, celui sur lequel il faut revenir lorsque qu'une forte perte de sens se produit, ce qui fût le cas avec le passage à la modernité avancée. Au Québec, durant la décennie des années 1970, les départements d'études des « arts et traditions populaires » (ethnologie) ont été envahis par les jeunes générations d'étudiants issus des nouveaux quartiers urbains et en quête d'authentique. Les séries télévisées les plus à la mode étaient celles mettant en vedette ce rural traditionnel. En France, rappelons le grand mouvement de retour à la nature après 1968, qui participe d'une crise de la civilisation urbaine moderne.

De manière plutôt inattendue, c'est du côté de l'écologie et de la nouvelle pensée sociale environnementaliste que va se former une autre identité de la ruralité. Celle-ci prend en compte les images survalorisantes qui circulent déjà et redonnent à la ruralité une signification forte, utilitariste et pratiquement post-moderne, soit la ruralité comme un environnement à protéger, à défendre ou comme un environnement capable de contribuer au ressourcement des populations urbaines qui vont s'y reposer dans le cadre des migrations pendulaires quotidiennes, hebdomadaires ou saisonnières, du tourisme vert et de ses nouvelles activités récréatives. La question de l'environnement qui prend une place prépondérante dans les débats sociaux actuels pèse lourdement dans les évolutions à venir des espaces ruraux. Car, dans les équations citadines habituelles

3. Nous faisons allusion ici au titre de la traduction française de l'ouvrage de Everett C. Hughes, Cantonville, une étude de l'urbanisation de la société québécoise par une monographie d'un anthropologue de l'École de Chicago. Cette monographie d'une ville moyenne en région rurale, véritable « boum town » de l'après-guerre, décrit bien cette rencontre des ruraux et des urbains et le processus culturel de l'urbanisation de la culture rurale. 
que nous révèlent les sondages d'opinion, l'environnement c'est la nature, et la nature c'est la campagne.

Cette préoccupation collective pour l'environnement fait en quelque sorte irruption dans l'espace rural et le fait apparaître sous un jour nouveau. Avec le déploiement de la modernité émerge une représentation de la ruralité comme un environnement ou des paysages qui sont des «biens publics » ou collectifs qui n'appartiennent pas seulement aux ruraux mais à toute la société. Il faut d'abord démontrer le caractère réducteur d'une telle équation. La campagne, par les actions séculaires d'aménagement des collectivités humaines, est loin d'être aussi «naturelle » qu'il y parait vue de loin, vue de la ville. De plus, avec une telle représentation sociale qui devient dominante, les populations urbaines se sentent légitimées de demander des comptes aux populations rurales sur la manière dont elles produisent des biens pour eux, notamment alimentaires. Il s'en suit un élargissement des dispositifs réglementaires concernant divers produits ruraux suite à des demandes urbaines le plus souvent mal comprises par les producteurs ruraux qui ont alors l'impression de se voir imposer des codes de conduite par des acteurs urbains par ailleurs mal informés des réalités rurales. Cette nouvelle représentation émergente de ruralité devient potentiellement porteuse d'incompréhension et de conflits de cohabitation tout autant qu'elle peut permettre de créer de nouvelles solidarités rurales/urbaines basées sur une meilleure compréhension des liens d'interdépendance entre ces deux mondes. On voit ainsi combien le discours social peut interpeller assez directement le discours politique.

\section{LE DISCOURS POLITIQUE}

Il ne faudrait pas s'attendre à ce que le discours politique soit très éloigné des deux précédents car le propre de celui-ci est de continuellement chercher et tenter de faire une synthèse entre discours savant (rationnel) et discours populaire (perceptions). Le discours politique est aussi, par définition, un discours réducteur. Par ailleurs, il faut aussi relier le discours politique à l'action car, comme on le constate souvent, le politique est souvent tenté de sublimer dans une inflation discursive son inaction sur le plan de l'action! Et cela est en bonne partie vrai avec la question rurale, objet de discours mais rarement objet d'une politique spécifique avec des objectifs clairs et des moyens d'intervention conséquents.

Selon nous, le discours politique à l'époque de la modernité naissante est un discours qui va dans le sens des idéologies dominantes de la ruralité. Il en résulte un discours fortement modernisateur qui identifie une tâche de l'État, celle de contribuer à « l'aménagement rural ». Avec le passage à la modernité avancée, le discours politique emboîte le pas et, dans le sillage du discours social, il professe son intention de développer des formules appropriées de « développement durable » des campagnes. Les velléités de modernisation font alors place à celles d'une préservation des territoires et des communautés rurales vues pratiquement comme des zones sinistrées ou des espèces en voie de disparition, nécessitant la sollicitude des pouvoirs publics pour assurer la pérennité de ces milieux socio-économiques fragilisés.

Le discours aménagiste de l’État (qui a connu son apogée durant les décennies 1960 et 1970) applique à la ruralité la même vision que dans d'autres domaines où les gouvernements se considèrent subitement légitimés d’intervenir dans de nombreuses dimensions de la vie sociale et économique. De plus, ce discours technocratique est un discours qui ne doute pas : il repose sur les dernières vérités de la démarche scientifique et technique, et propose des actions qui vont nécessairement produire les résultats escomptés en terme d'aménagement rural assurant aux campagnes un rattrapage pour rejoindre le train de la modernité qui est déjà en pleine course. Plusieurs images ont été proposées pour décrire ce modèle d'action de l'État. On a souvent parlé de l'État entrepreneur, qui va d'ailleurs se substituer un peu plus tard en un État accompagnateur, «facilitateur» ou encore partenaire dans l'ère de la modernité avancée où le discours étatique est moins certain de lui-même, beaucoup moins triomphaliste, comme en témoigne plusieurs politiques publiques rurales en Europe (les programmes LEADER), aux Etats-Unis (les Rural Par- 
tnerships) et au Québec (Politique Nationale de la Ruralité). Comme l’a bien montré Paul Houée, le discours sur la préservation des milieux ruraux, qui devient l'affaire de tout le monde et la sienne propre, inquiète bien des ruraux pour lesquels les campagnes ont besoin de véritables politiques de « développement rural », fussent-elles pensées selon la dernière version de la philosophie du développement durable.

\section{Conclusion : dépasser objectivation et constructivisme}

Dans le cadre de cette réflexion, nous ne prétendons pas, loin s'en faut, être parvenu à tenir entièrement notre promesse d'une authentique discussion entre ancrage empirique et constructions sociales des représentations au sujet des différentes ruralités québécoises dans la modernité avancée. L'exercice dialectique annoncé, et toujours à faire selon nous, s'arrête encore à la juxtaposition de deux réflexions distinctes : les appréciations statistiques permettant de saisir les visages nombreux et singuliers de la ruralité contemporaine d'un côté, l'évolution des représentations de ces mêmes réalités idéelles de l'autre.

Au moins, par analogie avec la géométrie, nous percevons clairement ces deux réflexions comme concourantes, c'est-à-dire qu'elle vont fatalement finir par se rencontrer, dialoguer et s'enrichir mutuellement. C'est ce que nous entendons par l'idée de dépasser l'opposition entre objectivation et constructivisme (observer, mesurer, modéliser les systèmes ruraux, y compris dans leur relation avec l'urbain, tout en analysant les systèmes de représentations qui en émanent et mobilisent les acteurs et l'action politique au sens large). Il y a toujours pertinence à se doter d'une typologie des ruralités contemporaines au Québec et cet exercice passerait à côté de l'essentiel si, en toute finalité, il ne prenait pas en compte les processus de construction de ses représentations.

En cela, pour nous, il apparaît de plus en plus que la notion de « développement territorial » constitue une perspective scientifique susceptible d'apporter un renouvellement de la compréhension du rôle et de l'influence réciproques tant des structures que des acteurs sur la formation et la recomposition des espaces socioéconomiques et politiques. Le développement territorial correspond à la fois à un courant de recherche en émergence, mais aussi à un foisonnement d'initiatives, surtout publiques, visant à mieux comprendre et maîtriser les facteurs qui déterminent les performances économiques d'ensembles territoriaux plus ou moins vastes. Ces facteurs sont d'ordre économique, culturel, politique, et liés aux caractéristiques des territoires où interviennent des acteurs sociaux.

\section{Bibliographie}

ASSOCIATION DES RURALISTES FRANÇAIS, 1998. - Journée régionale de l'ARF, Comment les ruraux vivent-ils et construisent-ils leur(s) territoire(s) aujourd'hui?, Toulouse, Maison de la recherche, Université de Toulouse Le Mirail, 287 p.

Bodiguel (M.), 1986. - Le rural en question, Paris, L'Harmattan, 183 p.

ÉPENDA (A.), 2003. - Les milieux ruraux québécois en restructuration : diagnostic, facteurs tangibles et intangibles de dévitalisation rurale et perspectives de développement local approprié, Thèse de doctorat, Université du Québec à Rimouski (disponible à : [www.uqar.qc.ca/crdt] dans la section «Études et rapports de recherche »), $341 \mathrm{p}$.

Frouws (J.), 1998. - « The Contested Redefinition of the Countryside. An Analysis of Rural Discourses in the Netherlands », Sociologia Ruralis, n 38 (1), p. 54-68.

JeAn (B.), Dionne (S.), Épenda (A.), Leblanc (P.), 2004. - « Une politique rurale pour quels territoires ruraux? La construction d'une typologie pertinente de la ruralité québécoise pour évaluer les retombées de la politique rurale », communication présentée dans le cadre de la section Développement régional 
du Congrès de l'Association canadienne-française pour l'avancement des sciences, mai 2004 (disponible à : [www.uqar.qc.ca/crdt] dans la section "Communications et conférences »), $20 \mathrm{p}$.

JEAN (B.), 2004. - « La question rurale. La place de la modernité avancée et la recomposition des systèmes ruraux au Québec », Les Carnets de l'Observatoire des rapports entre rural et urbain, UMR Dynamiques sociales et recomposition des espaces du CNRS, LADYSS, n² 2, janvier 2004, p. 5-31.

Jean (B.), 2003. - « La construction sociale de la ruralité », dans Poullaouec-Gonidec (P.), Paquette (S.), Domon (G.) (dir.), Le temps du paysage, Montréal, Les Presses de l'Université de Montréal, p. 105124.

Jean (B.), Chevalier (J.), Klein (J.-L.), Sztokman (N.) (dir.), 1991. - De la Loire au Saint-Laurent. Des régions rurales face aux recompositions socio-territoriales, Rimouski-Chicoutimi-Nantes, Co-édition GRIDEQ/GRIR/CNRS, 1991, 340 p.

JEAN (B.), 1989. - «La question rurale : la ruralité et sa sociologie », Recherches Sociologiques, n 20 (3), Louvain-la-Neuve, Centre de sociologie rurale et urbaine, Université Catholique de Louvain, numéro thématique intitulé « Sociologie rurale ou sociologie du rural ? », p. 287-309.

Kalaora (B.), 1998. - Au-delà de la nature, l'environnement, Paris, L'Harmattan, 199 p.

Mathieu (N.), Jollivet (M.), 1989. - Du rural à l'environnement. La question de la nature aujourd'hui, Paris, ARF/L'Harmattan, $354 \mathrm{p}$.

Perrier-Cornet (P.), 2002. - Les espaces ruraux dans la société urbanisée, DATAR, Éditions l'Aube, 280 p.

Poulle F., Gorgeu (Y.), 1997. - Essai sur l'urbanité rurale, Paris, Syros, 172 p.

VACHON (B.) (dir.), 1991. - Le Québec rural dans tous ses états, Textes produits aux États Généraux du monde rural, Montréal, Éditions du Boréal, 309 p.

Cet article a été reçu le 30 juin 2006 et définitivement accepté le 15 janvier 2007. 
